\title{
Allocating hearts
}

\author{
Nicholas G. Smedira, MD
}

See related article on page 925 .

From the Cleveland Clinic Foundation, Cleveland, Ohio.

Received for publication Nov 11, 2005; accepted for publication Nov 18, 2005.

Address for reprints: Nicholas G. Smedira, MD, Cleveland Clinic Foundation, 9500 Euclid Avenue Desk F24, Cleveland, OH 44195 (E-mail: smedirn@ccf.org).

J Thorac Cardiovasc Surg 2006;131:775-6

$0022-5223 / \$ 32.00$

Copyright $\odot 2006$ by The American Association for Thoracic Surgery

doi:10.1016/j.jtcvs.2005.11.032

$\Lambda$ s of August 5, 2005 there were 96,189 patients awaiting organ transplantation. During 2004 only 27,036 transplants were performed, highlighting a growing disparity between patients listed and organs available. The United Network for Organ Sharing (UNOS) is charged with coordinating the sharing of organs to ensure fairness and optimal utilization, and they also monitor the outcomes of all listed and transplanted patients. This wealth of information, which is accessible to the medical community and patients, was analyzed by Dr. Mokadam and colleagues, ${ }^{1}$ and as a result, they have raised concerns about the welfare of patients listed as status 2 (not inotrope-dependent, usually at home; patients listed as status 1A are hospitalized on devices or inotropes and patients listed as status 1B are on inotropes) during their prolonged wait for a heart transplant. Of the 1265 patients analyzed, 30\% deteriorated and $10 \%$ died. Before responding to these concerns, I would like to take a closer look at how patients are doing while waiting for a heart transplant.

Since 1994, there has been a dramatic and unanticipated decline in the annual number of heart transplants performed in the United States. As reported by UNOS, during the past decade, heart transplants have been declining at a rate of $1 \%$ to $2 \%$ per year from a peak of 2528 in 1995 to 2016 in 2004. Arguing against donor shortages as the cause, the number of patients listed annually also declined from a peak of 4079 in 1998 to 2802 in 2002. There has also been a shortening of the aggregate waiting time despite news to the contrary. In Mokadam's review the median wait time for patients listed as status 2 was 406 days.

This information is accurate but somewhat misleading. UNOS presents times as "time to transplant" and "waiting time before transplant." Time to transplant includes active and inactive time; waiting time is only active time. A patient may be inactivated for many reasons - too sick (early after left ventricular assist device [LVAD] insertion), too well, psychosocial, and personal-and physicians are reluctant to remove the patients from the list because they will lose their accumulated time on the list. The number of patients classified as "temporarily inactive" at the end of the calendar year increased over the decade from $33 \%$ to $48 \%$ of patients listed. Figure 1 shows the time to transplant with patients listed as status 2 waiting substantially longer; Figure 2 shows a substantial reduction in waiting times when only active time is considered. Currently UNOS does not tabulate the reasons for inactive listing or which status level is more commonly inactive. It makes little sense to me to discuss waiting times unless this is when you are actively looking for a donor organ for the patient.

Great strides have been made in reducing the mortality of the patients on the waiting list (Figure 3). For all statuses listed, death rates have declined, with patients listed as status 2 having the lowest risk of dying while awaiting transplant. Two commonly offered justifications for transplanting patients listed as status 2 are that they do better after transplantation compared with patients listed as status 1 and they do poorly if they deteriorate and need urgent mechanical support or transplantation. The available data refutes this. The unadjusted 1-year survival is higher for patients listed as status 2 when compared with patients listed as status $1 \mathrm{~A}$ ( $88 \%$ vs $81 \%$, respectively). However, when adjusted for other covariates, status at listing does not impact survival after transplantation. ${ }^{2}$ Using the same UNOS data as Mokadam did, Jimenez and colleagues found that deterioration to status $1 \mathrm{~A}$ or $1 \mathrm{~B}$ from status 2 did not impact survival after transplantation. ${ }^{3}$

Although the information presented in their brief communication is true, it does not accurately reflect the trends I have outlined from the UNOS database when analyzed on the basis of actively listed patients and using death rates rather than simple proportions. Two concerns were raised by the authors: it is ethically unjustifiable to conduct a prospective randomized trial of medical therapy versus transplantation in status 2 
Time to Transplant

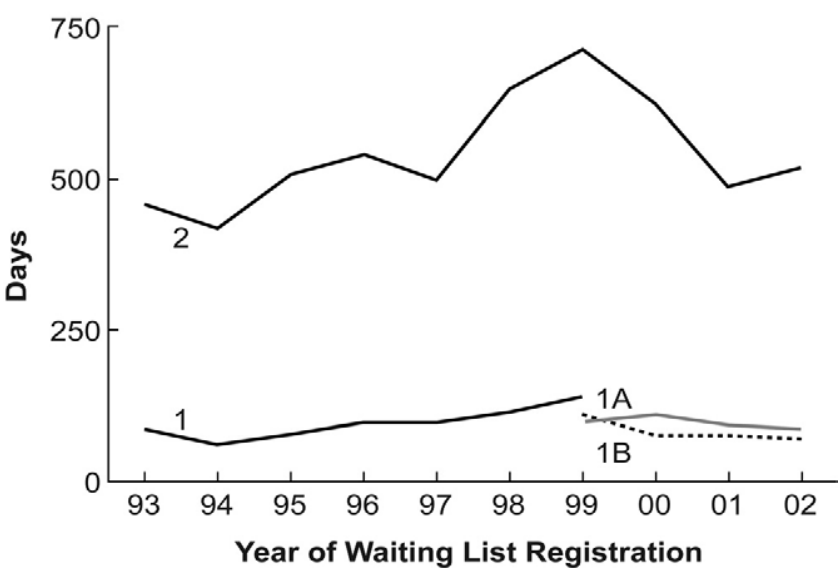

Figure 1. Time to transplantation for all statuses. patients and it is premature to divert organs from local patients listed as status 2. UNOS allocates hearts locally (in the city or region of the donor hospital) first, with patients listed as status $1 \mathrm{~A}, 1 \mathrm{~B}$, and 2 considered, and then the hearts would be allocated to patients listed as status $1 \mathrm{~A}$ or $1 \mathrm{~B}$ within 500 , then 1000, nautical miles of the donor hospital. In Ohio we have a sharing arrangement that supersedes this, and all hearts donated in Ohio are shared among transplant centers and allocated by status-1A before 1B before 2 . UNOS has proposed that the first offer would be to patients listed as status 1A or 1B within 500 miles of the donor hospital. To comprehend how many centers this would impact, as an example with the Cleveland Clinic at the center of a circle with a 500-mile radius and a flying time of approximately 90 minutes, a donor in Cleveland could be shared with centers as far away as Augusta, Maine; Savannah, Georgia; Memphis, Tennessee; St Louis, Missouri; and St Paul, Minnesota. From the data I have pre-

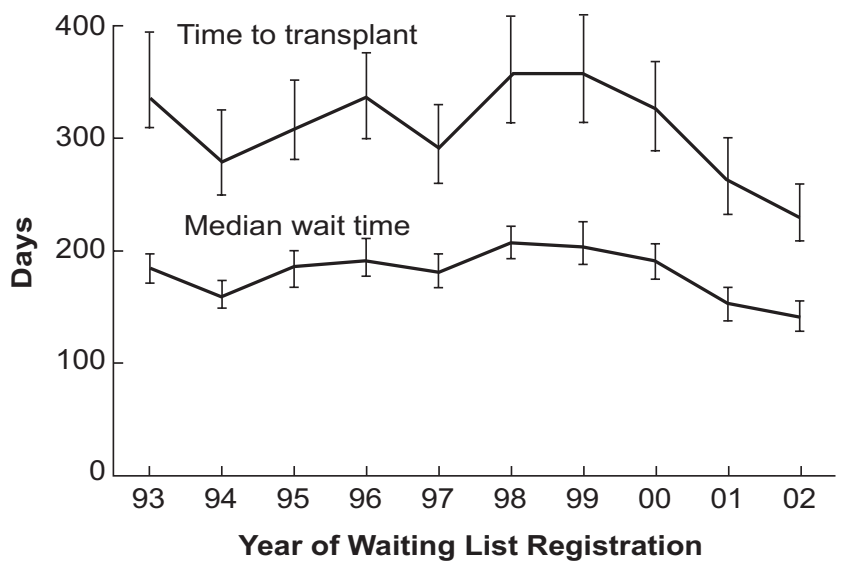

Figure 2. Time to and waiting time until transplantation.
Annual Death Rates Per 1,000 Patients Year at Risk

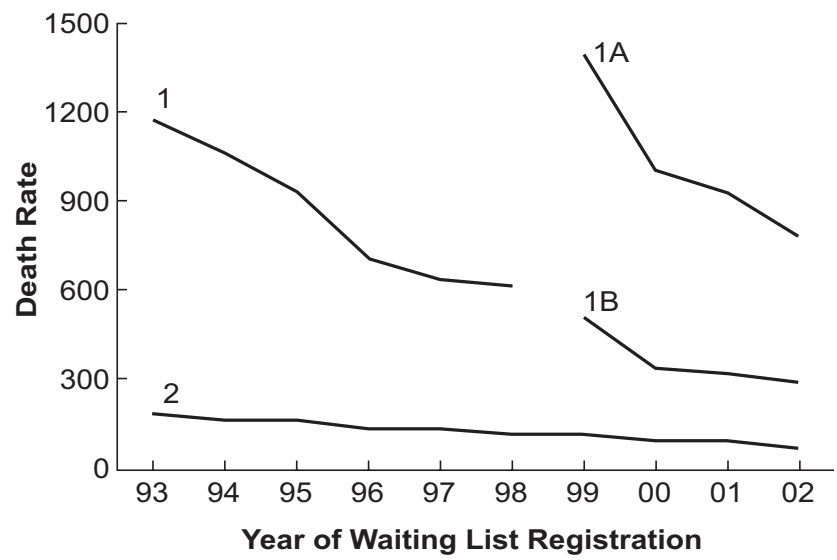

Figure 3. Mortality rates for all statuses.

sented and our experience with an in-state sharing agreement, this makes a great deal of sense. Transplant News reported on November 30, 2005 that this allocation scheme was adopted by the UNOS Board of Directors and they predict a $7 \%$ reduction in pretransplant deaths in both adults and pediatrics. ${ }^{4}$

Should we consider randomizing patients listed as status 2? I have not seen a protocol to comment on. The reason this question is being asked by transplant physicians is because at 1 and 2 years after being listed as status 2, 50\% and 20\% of patients, respectively, are alive without transplantation or deterioration. Rather than a trial, an effort should be made to understand why some patients remain clinically stable after listing.

The transplant community should take great pride in the advances in cardiovascular medicine that have reduced the number of patients needing to be actively listed, reduced the waiting time for those listed, improved the safety of patients awaiting transplantation, and developed an allocation scheme that accurately identifies patients at greatest risk of dying. Further refinement of medical therapies and modifications of the allocation system should be implemented if they reduce the aggregate mortality for all patients requiring a heart transplant

The author thanks Drs Randall C. Starling and James B. Young for their assistance in the preparation of this editorial.

\section{References}

1. Mokadam NA, Ewald GA, Damiano Jr. RJ, Moazami N. Deterioration and mortality among patients with United Network for Organ Sharing status 2 heart disease: Caution must be exercised in diverting organs. J Thorac Cardiovasc Surg. 2006;131:925-6.

2. Taylor DO, Edwards LB, Boucek MM, et al. Registry of the International Society for Heart and Lung Transplantation: Twenty-second Official Adult Heart Transplant Report-2005. J Heart Lung Transplant. 2005;24:945-55.

3. Jimenez J, Edwards LB, Higgins R, et al. Should stable UNOS status 2 patients be transplanted? J Heart Lung Transplant. 2005;24:178-83.

4. Warren J. New heart allocation scheme ok'd by OPTN/UNOS Board will decrease waitlist deaths for adult, pediatrics. Transplant News. 2005;15:4. 